\title{
Ocorrência e autoecologia de Acanthoceras zachariasii (Brun) Simonsen (Chaetocerales, Coscinodiscophyceae) na região centro-norte do Brasil
}

\author{
Raquel Rezende de Morais ${ }^{1,6}$, Ina de Souza Nogueira², L. Felipe Artigas 3 , \\ Elizabeth Cristina Arantes de Oliveira Miranda ${ }^{4}$ e Weliton José da Silva ${ }^{5}$
}

Recebido: 11.03.2015; aceito: 5.10.2015

\begin{abstract}
Occurrence and autoecology of Acanthoceras zachariasii (Brun) Simonsen (Chaetocerales, Coscinodiscophyceae) in the Central-Northern Brazil). In this study we presented the structural and ultrastructural characterization of Acanthoceras zachariasii (Brun) Simonsen, as well as its geographical distribution in the Amazon River Basin and Tocantins-Araguaia River Basin, Brazil, and provided the lectotypification of the taxon. We analyzed 454 phytoplanktonic samples, but the occurrence of A. zachariasii was recorded in only 14 of them. Of the total samples collected along the two basins, A. zachariasii was recorded only in $3.08 \%$, which demonstrates the rarity of this taxon. This species was registered in lentic environments, with mesotrophic to eutrophic conditions, and $\mathrm{pH}$ varying from slightly acid to alkaline. Keywords: Amazon River Basin, Tocantins-Araguaia River Basin, Attheya zachariasii, Acanthoceras magdeburgense, lectotypification
\end{abstract}

RESUMO - (Ocorrência e autoecologia de Acanthoceras zachariasii (Brun) Simonsen (Chaetocerales, Coscinodiscophyceae) na região centro-norte do Brasil). Apresenta-se a caracterização estrutural e ultraestrutural de Acanthoceras zachariasii (Brun) Simonsen, bem como, a distribuição geográfica da espécie nas bacias Amazônica e do Tocantins-Araguaia, Brasil, além da lectotipificação do táxon. Foram analisadas 454 amostras fitoplactônicas, sendo registrada a ocorrência de $A$. zachariasii em apenas 14. Do total de amostras coletadas nos estudos das duas bacias, apenas em 3,08\% foram registrados representantes de A. zachariasii, demonstrando quão rara é a frequência de ocorrência do táxon. A espécie ocorreu em ambientes lênticos, mesotróficos a eutróficos, com $\mathrm{pH}$ variando de levemente ácidos a alcalinos.

Palavras-chave: Bacia Amazônica, Bacia do Tocantins-Araguaia, Attheya zachariasii, Acanthoceras magdeburgense, lectotipificação

\section{Introdução}

Acanthoceras zachariasii (Brun) Simonsen é caracterizada por apresentar frústula pouco silicificada, retangulares em vista pleural, com um espinho tubular em cada ângulo da valva (Round et al. 1990). A fragilidade da frústula dificulta a preservação e observação do táxon, uma vez que no processo de limpeza por oxidação e fixação o organismo pode ser destruído (Tremarin et al. 2015). Provavelmente seja este o motivo dos poucos registros desse táxon para o Brasil e para o mundo.

Apesar dos poucos registros no país, A. zachariasii possui distribuição ampla, cosmopolita, porém a espécie, em geral, apresenta populações pouco expressivas (Beaver 1981, Cărăus 2012, Edlund \& Soermer 1993, Ferrario et al. 1992, Genkal \& Chekryzheva 2011, Gupta \& Singh 1985, Huber-

1. Universidade Federal de Goiás, Instituto de Ciências Biológicas, Programa de Pós-graduação em Biodiversidade Vegetal da UFG, Laboratório de Análise e Gerenciamento Ambiental de Recursos Hídricos, Goiânia, Goiás, Brasil

2. Universidade Federal de Goiás, Instituto de Ciências Biológicas, Programa de Pós-graduação em Biodiversidade Vegetal da UFG, Laboratório de Análise e Gerenciamento Ambiental de Recursos Hídricos, Goiânia, Goiás, Brasil

3. Laboratoire d'Océanologie et Géosciences, CNRS, Université du Littoral Cô te d'Opale, 32 avenue Foch, 62930 Wimereux, France

4. Universidade de Brasilia, Instituto de Geociências, Laboratório de Limnologia, Brasília, Distrito Federal, Brasil

5. Universidade Federal de Goiás, Instituto de Ciências Biológicas, Programa de Pós-graduação em Biodiversidade Vegetal da UFG, Laboratório de Análise e Gerenciamento Ambiental de Recursos Hídricos, Goiânia, Goiás, Brasil

6. Autor para correspondência: raquelmorais3@hotmail.com 
Pestalozzi 1942, Hustedt 1930, Krammer \& LangeBertalot 1991, Lepistö 1999, Lepistö et al. 2004, Lozo et al. 2013, Napiórkowska-Krzebietke et al. 2011, Negro \& De Hoyos 2005, Nõges \& Viirret 2001, Rivera 1974, Schagerl et al. 2009, Shirata 1986, Shirata \& Valente-Moreira 1987, Sinada \& Abdel Karim 1984, Townsend 2006, Train \& Rodrigues 2004, Tremarin et al. 2015).

Para o Brasil A. zachariasii é registrada por Shirata (1986), Shirata \& Valente-Moreira (1987), Train \& Rodrigues (2004) e Tremarin et al. (2015) que apresentam a distribuição geográfica no Estado do Paraná. No estado do Mato Grosso do Sul esta espécie foi citado por Brasil das Águas (Moss \& Moss 2007) para o município de Rio Verde. Este táxon ainda não havia sido registrado para o Estado de Goiás e região Norte do País.

A taxonomia de A. zachariasii com base em dados morfológicos estruturais é detalhada por e Edlund \& Wynne (1996) \& Ferrario et al. (1992), enquanto que caracterizações ultraestruturais são fornecidas por Round et al. (1990). Esta espécie ocorre em corpos d'água alcalinos com elevada transparência (Edlund \& Stoermer 1993, Townsend, 2006)s oligotróficos a hiper-eutróficos (Edlund \& Stoermer 1993, Gupta \& Singh 1985, Hustedt 1930, Krammer \& LangeBertalot 1991, Lepistö 1999, Lepistö et al. 2004, Napiórkowska-Krzebietke et al. 2011, Townsend 200,).

Acanthoceras zachariasii $[\equiv$ Atteya zachariasii Brun] ainda não foi tipificada. A ausência da indicação de tipo nomenclatural é muito comum nas descrições de diatomáceas e muitas outras algas e plantas descritas originalmente até a metade do século XX. Esse fato ocorre devido a indicação do tipo ter se tornado requerimento essencial somente a partir de 1958 de acordo com o Art. 40.1 do Código Internacional de Nomenclatura para algas, fungos e plantas (CIN, McNeill et al. 2012). Para casos como esse, o Art. 9.11 do CIN permite a indicação de um lectótipo (i.e., indicação de um tipo a partir de espécimes ou ilustrações originais), ao qual o nome da espécie estará permanentemente vinculado (McNeill et al. 2012).

O objetivo desse estudo é apresentar a ocorrência de A. zachariasii para as regiões hidrográficas do Tocantins-Araguaia e Amazônica, descrever a estrutura e ultraestrutura de representantes dessa espécie para estas regiões, e realizar a lectotipificação desse táxon .

\section{Material e métodos}

O material estudado é proveniente de rios e lagoas de várzea das regiões hidrográficas amazônica e do Tocantins-Araguaia (figura 1), coletadas entre os anos de 2000 e 2012. Amostragens para análise qualitativa foram efetuadas a partir da filtragem de água na subsuperfície dos sistemas utilizando rede de plâncton de $25 \mu \mathrm{m}$ de abertura de malha. Estas amostras foram fixadas com solução de Transeau no momento da coleta (Bicudo \& Menezes 2006) e depositadas no Herbário UFG. Amostragens para análise quantitativa foram realizadas por meio de coleta de água bruta na subsuperfície dos sistemas aquáticos, fixadas com lugol acético (Bicudo \& Menezes 2006). De um total de 454 amostras analisadas, somente 14 foram retidas para este estudo nas quais foi registrada a ocorrência de A. zachariasii (tabela 1).

$\mathrm{Na}$ Bacia Amazônica, as variáveis $\mathrm{pH}$, condutividade, temperatura da água, e turbidez, foram aferidas em campo através da sonda multiparâmetros YSI modelo 6820-V2. Análises de nutrientes (i.e., nitrito, nitrato e ortofosfato) foram realizadas de acordo com Grasshoff (1983) na coleta realizada na bacia Amazônica. Na Bacia Tocantins-Araguaia, valores de $\mathrm{pH}$, condutividade, temperatura da água, e turbidez foram aferidas em campo através de sonda multiparâmetros Horiba U21. Os procedimentos de análises de nutrientes foram efetuados segundo métodos padronizados (APHA 2005). Os valores das variáveis limnológicas são registrados na tabela 2 . A caracterização dos estados tróficos dos ambientes aquáticos das bacias amazônica e Tocantins-Araguaia foram de acordo com OECD (1982).

As amostras para o estudo de ultraestrutura foram lavadas sucessivamente em água destilada, preparadas em lamínulas circulares de $18 \mathrm{~mm}$ e desidratadas em temperatura ambiente. Posteriormente, as lamínulas foram fixadas com fita de carbono em suportes de alumínio e recobertas com ouro paládio (Ferrario et al. 1992).

Os espécimes foram analisados em microscópio óptico (MO) Zeiss Axioscop 40, equipado com captura de imagem Axiocam HRc e o sistema Axiovision em 400 ou 1200 vezes de aumento. A análise ultraestrutural foi realizada através de microscópio eletrônico de varredura (MEV) Jeol modelo JSM 6610 , operado a $7 \mathrm{kV}$ e a distância de $11 \mathrm{~mm}$. O enquadramento sistemático e a terminologia para caracterização estrutural e ultraestrutural da frústula foi baseada em Round et al. (1990). 
A quantificação do fitoplâncton foi efetuada em microscópio invertido Zeiss Axiovert 25, sendo a densidade fitoplanctônica estimada segundo Utermöhl (1958) e expressa em ind. $\mathrm{mL}^{-1}$. O tempo de sedimentação das amostras nas câmaras de contagem seguiu o proposto por Margalef (1983). A contagem foi feita em campos distribuídos aleatoriamente (Uhelinger 1964) e os organismos foram quantificados com base na curva de rarefação de espécies (Bicudo 1990) ou até atingir 100 indivíduos da espécie mais abundante, de modo que o erro da contagem seja inferior a $20 \%$ e o nível de significância da ordem de 95\% (Lund et al. 1958).

\section{Resultados e Discussão}

Acanthoceras zachariasii (Brun) Simonsen, Bacillaria 2: 55, 1979 EAtheya zachariasii Brun, Forschungsber. Biol. Stat. Plön 2: 53, Fig. 1: 11a, 1894. Tipo: ALEMANHA. EsLÉSviCo-Holsácia:
Plön, zona limnética dos Lagos Grande e Pequeno de Plön [Lectótipo, designado aqui: O espécime ilustrado por Brun (1894, Fig. 1: 11a), reproduzido como a Figura 2 deste trabalho]. = Acanthoceras magdeburgense Honigm., Arch. Hydrobiol. 5: 77, Fig. 2:a, 1909.

Obs. Sugere-se que se faça uma discussão mais detalhada da lectotipificação do táxon, aqui proposta, pois aparece solta no trabalho

Figuras 2-7

Caracterização - Células solitárias, frústulas cilíndricas, delicadas, retangulares em vista pleural (figuras 3,4); comprimento (sem o espinho): 32,0-34,5 $\mu \mathrm{m}$, largura: 6,4-15,0 $\mu \mathrm{m}$; valvas bicônicas conectadas por uma ponte, ornadas com dois espinhos tubulares retos, um em cada ângulo de valva (figuras 3-6), espinhos: 20,4-22,6 $\mu \mathrm{m}$; aréolas irregulares distribuídas longitudinalmente, 1-3 fileiras formadas por conjuntos de aréolas, irradiando na valva no sentido da caliptra ou arranjadas irregularmente na base da valva, 48-57

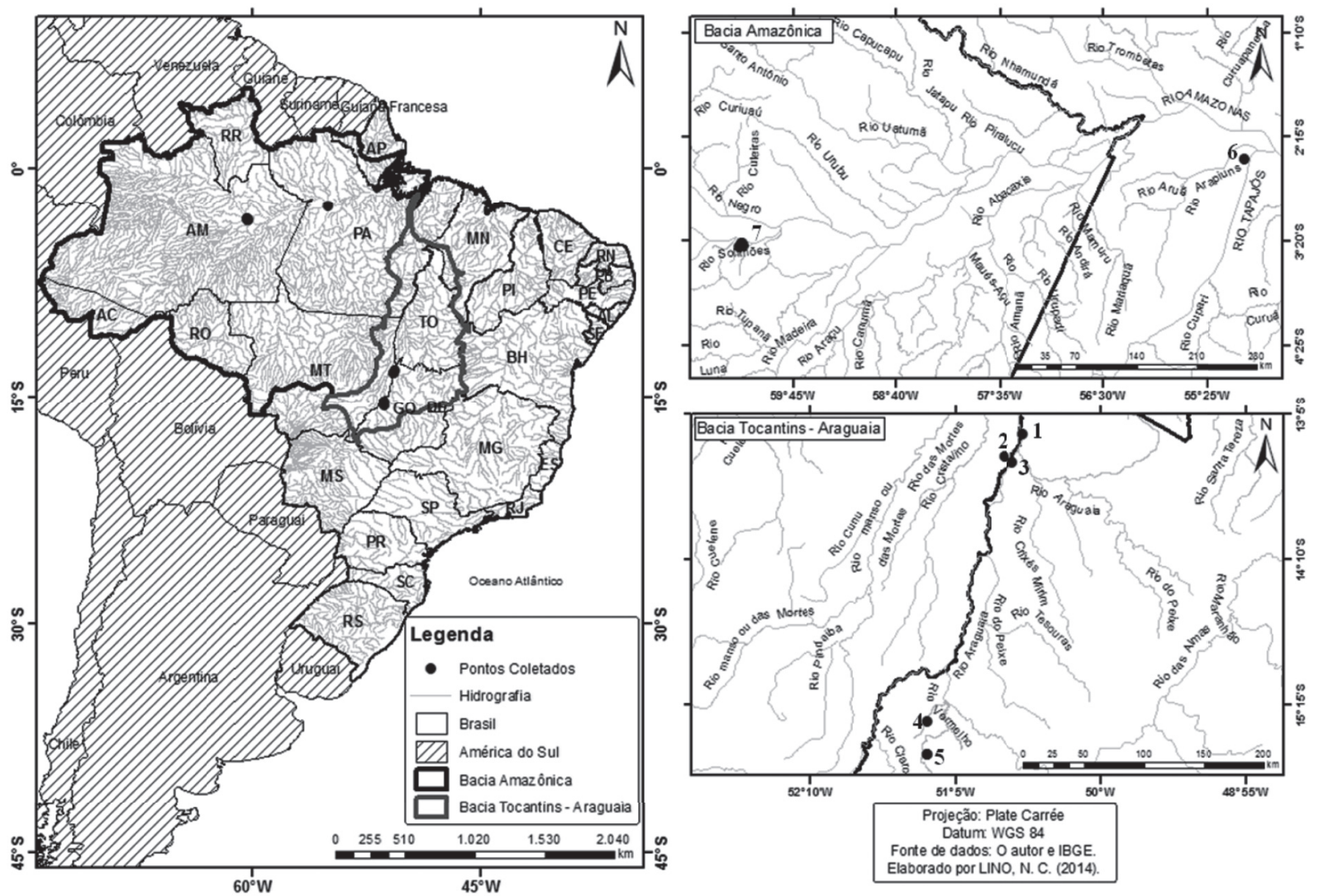

Figura 1. Mapa indicando as localidades de ocorrência de Acanthoceras zachariasii (Brun) Simonsen nas regiões Norte e Centro-Oeste do Brasil.

Figure 1. Map indicating the localities of occurrence of Acanthoceras zachariasii (Brun) Simonsen in the North and Central-Western regions of Brazil. 


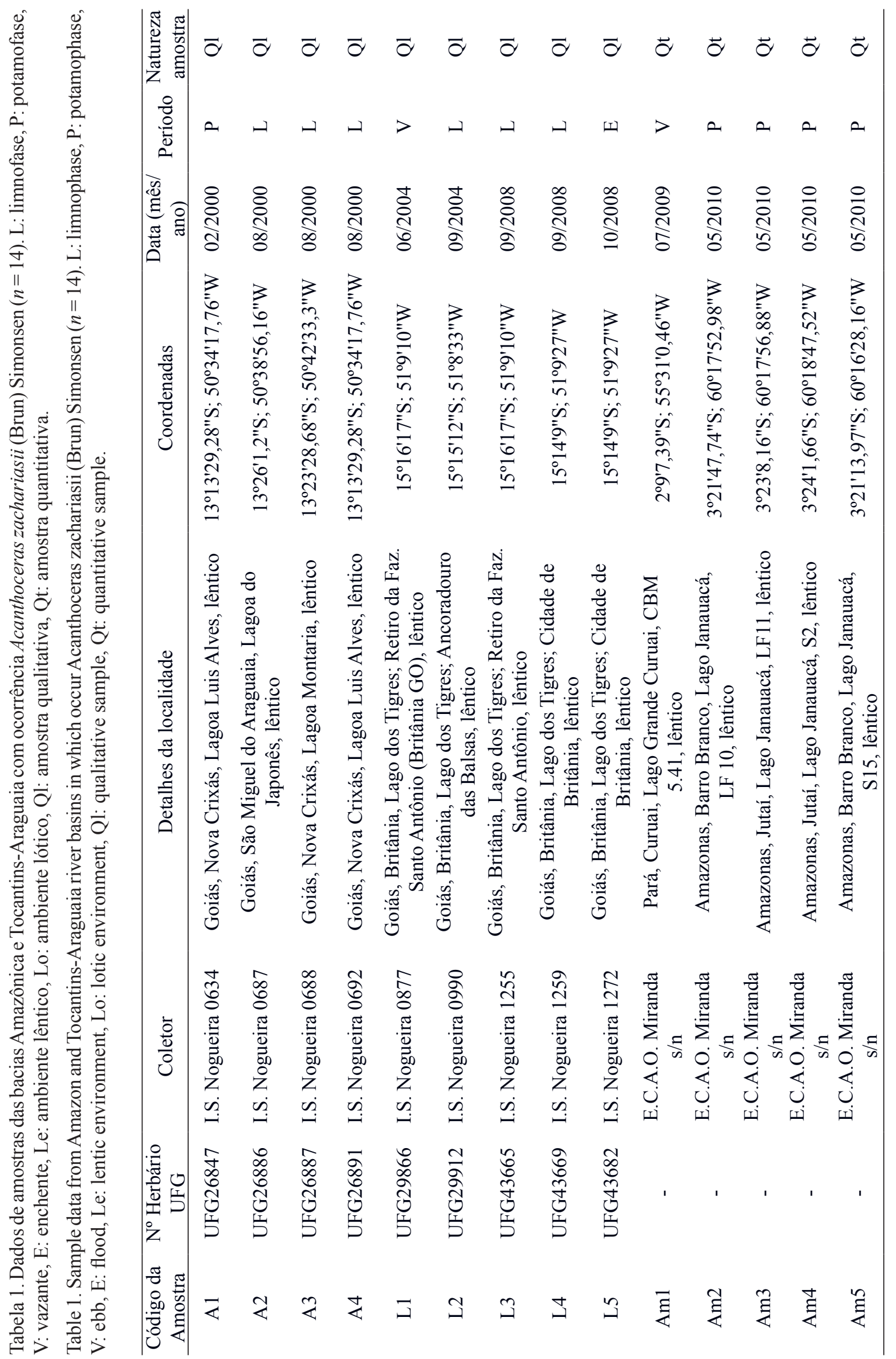


aréolas em $10 \mu \mathrm{m}$ (figuras 5,6); cíngulo formado por bandas imbricadas, 4-6 bandas em $10 \mu \mathrm{m}$, providas de aréolas irregulares, dispostas em linhas próximo da junção da banda e disposição irregular na região mediana, 70-75 aréolas em $10 \mu \mathrm{m}$ (figuras 5, 7); 4 cloroplastos discoides, pequenos e axiais (figura 3 ).

Material examinado: BRASIL. GoIÁs: Nova Crixás, Lagoa Luís Alves, II-2000, I.S. Nogueira 634 (UFG26847); VIII-2000, I.S. Nogueira 692 (UFG26891); Nova Crixás, Lagoa Montaria, VIII-2000, I.S. Nogueira 688 (UFG26887); São Miguel do Araguaia, Lagoa do Japonês, VIII-2000, I.S. Nogueira 687 (UFG26886); Britânia, Retiro da Faz. Santo Antônio,VI-2004, I.S. Nogueira 877 (UFG29866); XIX-2008, I.S. Nogueira 1255 (UFG43665); Britânia, Ancoradouro da Balsa, XIX-2004, I.S. Nogueira 990 (UFG29912); Britânia, Cristo Redentor, XIX-2008, I.S. Nogueira 1259 (UFG43669), X-2008, I. S. Nogueira 1272 (UFG43682); PARÁ: Curuai, Lago Grande Curuai, 7-VII-2009, Projeto CARBAMA CBM5.41; AMAZÔNIA: Barro Branco, Lago Janauacá, LF10, S15, V-2010, E. C. A. O. Miranda s.n.; Jutaí, Lago Janauacá, LF11, S2, V-2010, E. C. A. O. Miranda s.n.

Os representantes registrados na região centronorte do Brasil foram menores do que aqueles do material original, tanto com relação ao comprimento da frústula sem os espinhos $(32,0-34,5 \mu \mathrm{m}$ vs. 60$100 \mu \mathrm{m})$ quanto em relação à largura $(6,4-15,0$ $\mu \mathrm{m}$ vs. 15-20 $\mu \mathrm{m})$ (Brun 1894). Espécimes com valores de comprimento similares aos do material original $(56,5-73,4 \mu \mathrm{m})$, porém mais estreitos $(6,1$ $13,5 \mu \mathrm{m})$ similarmente ao material do centro-norte brasileiro, foram encontrados em reservatórios do Sul do país (Tremarin et al. 2015). Entretanto, uma grande variabilidade dos caracteres morfométricos é registrada em outros trabalhos os quais, em geral, apresentam comprimento variando de 12-60 $\mu \mathrm{m}$ e largura de 8,5-40 $\mu \mathrm{m}$ (tabela 3).

Comparado ainda aos espécimes de sistemas aquáticos da região Sul do Brasil, representantes de A. zachariazii do centro-norte apresentaram valores similares de densidade de aréolas nas valvas (48-57 vs. 50-55 aréolas em $10 \mu \mathrm{m}$ ) e nas bandas do cíngulo (70-75 vs. 70 aréolas em $10 \mu \mathrm{m}$ ), e menor densidade de bandas (4-6 vs. 9-14 bandas em $10 \mu \mathrm{m}$ ) (Tremarin et al. 2015). Contudo, esta última característica concorda com aquelas registradas por Ferrario et al. (1992) e Rivera (1974).
A grande variabilidade das características morfológicas de representantes identificados como A. zachariazii distribuídos em diferentes localidades do mundo pode sugerir que este táxon trata-se de um complexo de espécies crípticas, semicrípticas ou pseudocrípticas. Casos similares têm sido registrados para outros táxons de diatomáceas, principalmente grupos de penada (Li et al. 2010, Kermarrec et al. 2013). Por outro lado, pouco ainda se conhece a respeito da plasticidade fenotípica e da confiabilidade de caracteres na delimitação de táxons em diatomáceas (Mann 1999, Mann \& Evans 2007). Estudos dessa natureza são ainda escassos e requerem, inclusive, análises filogenéticas utilizando dados moleculares a partir de abordagens polifásicas (Mann \& Evans 2007, Zimmermann et al. 2011, Abarca et al. 2014).

Assim como muitas diatomáceas, originalmente descritas antes da primeira metade do século XX, Acanthoceras zachariazii não foi tipificada. A indicação de tipos nomenclaturais tornou-se requerimento indispensável para a validação de propostas de nomes de táxons abaixo do nível de família a partir de 1958, conforme estabelecido no Art. 40.1 do CIN (McNeill et al. 2012)(McNeill et al. 2012). Tipos são essenciais para a fixação do nome de um dado táxon. Caso existam espécimes ou ilustrações originais do táxon, o CIN permite a indicação de um lectótipo a partir da escolha de um deles (Art. 9.12, McNeill et al. 2012). O CIN estabelece ainda no Art. 9.1 Nota 1, que se o autor do táxon utilizou um só elemento (i.e., espécime ou ilustração), este deve ser aceito como holótipo. Tal afirmação é, algumas vezes, interpretada equivocadamente quando alguns pesquisadores consideram o termo "utilizou" como sinônimo de "publicou" ou "representou". A referida nota, no entanto, refere-se a casos em que descrições são baseadas comprovadamente em um único elemento ou ilustração, como pode ser constatado nos Exemplos 1 e 2 do Art. 9.1 Nota 1 fornecidos pelo CIN (McNeill et al. 2012). Esse é o caso de Schizonema dillwynii C.Agardh, em que Agardh (1824)(1824) deixa claro ter baseado a descrição desse táxon na ilustração de Conferva foetida Villars sensu Dillwyn (Dillwyn 1809, fig. 104, Jurg. 7)(1809, fig. 104, Jurg. 7) non C. foetida Villars (1789)(1789).

Espécimes originais de $A$. zachariazii não foram encontrados em instituições onde estão depositados materiais de J. Brun (i.e., herbários AWH, G, e PC, e no Lycee Blaise-Cendrars, Suíça). Contudo, o fato de não terem sido encontrados espécimes originais não 


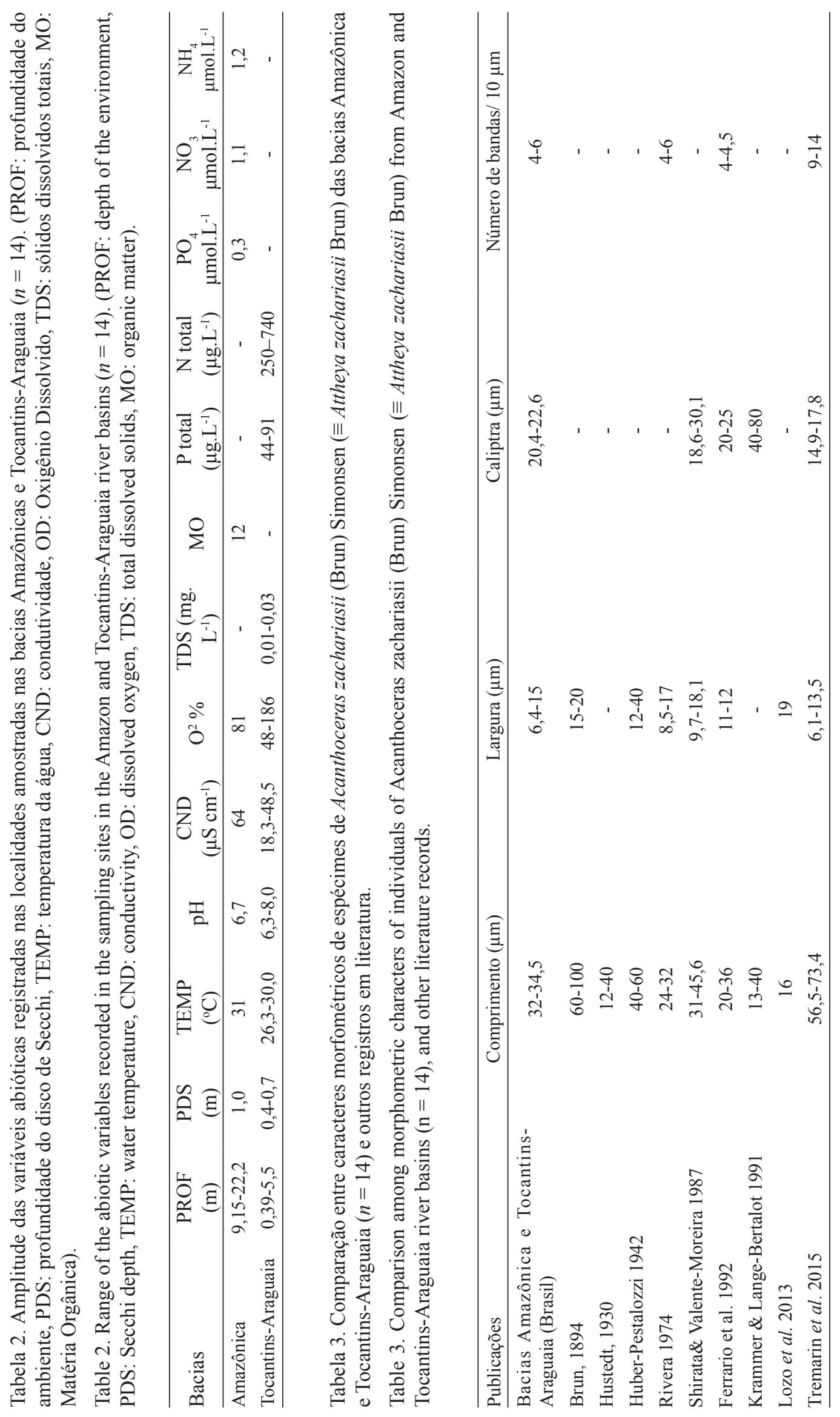


implica, necessariamente, que foi utilizado somente um elemento, tal como uma ilustração publicada pelo próprio autor, para a descrição do táxon (J. McNeill, N. Turland, S. Knapp e J. Prado, comunicação pessoal). Em exemplo de situação similar foi a lectotipificação de Placoneis placentula (Ehrenberg) Mereschkowsky, para a qual não foram encontrados espécimes originais e, portanto, foi utilizada ilustração do táxon (Jahn 2004)(Jahn 2004). Dessa forma, a lectotipificação de $A$. zachariazii utilizou designou o único material original disponível até então, i.e., a ilustração publicada pelo autor. Esta lectotipificação não será inefetivada nem mesmo se foram encontrados em algum momento os espécimes originais, já que não está em qualquer desacordo com o protólogo (Art. 9.19, McNeill et al. 2012).

O gênero Acanthoceras Honigmann nom. cons. foi proposto por Honigmann (1910)(1910) com apenas dois táxons, A. magdeburgense [magdeburgensis] Honigmann var. magdeburgense (tipo genérico) e A. magdeburgense var. latum [lata] Honigmann. Contudo, apesar de ser amplamente utilizado, o nome do gênero foi, durante algumas décadas, tratado como ilegítimo. Tal ilegitimidade deu-se por Acanthoceras

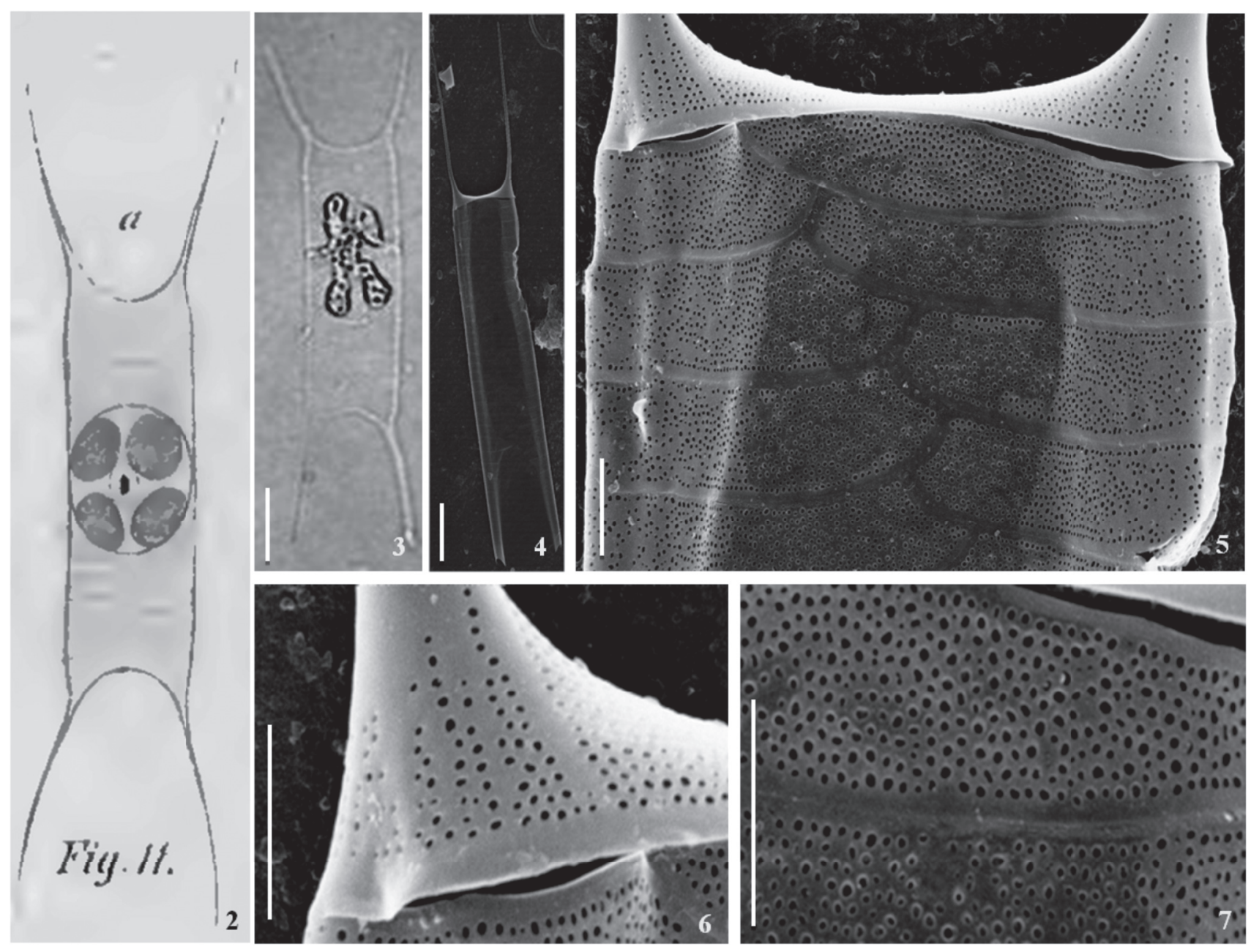

Figuras 2-7. Acanthoceras zachariasii (Brun) Simonsen. 2. Lectótipo (designado aqui) a partir da Figura 1: 11a de Attheya zachariasii Brun, provida por Brun (1894). 3. Vista pleural em microscopia óptica. 4-6. Vista pleural em microscopia eletrônica de varredura. 5. Valva bicônica, destacando a base das caliptras, e bandas do cíngulo imbricadas e ornamentadas por aréolas irregulares. 6. Detalhe das aréolas na valva, formando pequenas fileiras de grupos de pontos da margem valvar em direção à caliptra. 7. Detalhe das aréolas na banda do cíngulo. Escala: Figures 3, $4=10 \mu \mathrm{m}$; Figures 5-7 = $2 \mu \mathrm{m}$.

Figures 2-7. Acanthoceras zachariasii (Brun) Simonsen. 2. Lecotype (designated here) from the Figure 1: 11a of Attheya zachariasii Brun, provided by Brun (1894). 3. Pleural view in light microscopy. 4-6. Pleural view in scanning electron microscopy. 5. Biconical valve, highlighting the base of the calyptras, bands of the cingulum, imbricated and ornamented with irregular areolae. 6 . Detail of the areaolae in the valve, forming small lines of groups of dots from the margin of the valve towards the calyptra. 7. Detail of the areolae in the bands of the cingulum. Scale bar: Figures 3, $4=10 \mu \mathrm{m}$; Figures 5-7 = $2 \mu \mathrm{m}$. 
Honigmann tratar-se de um homônimo posterior de Acanthoceras Kützing (1842)(1842), um gênero de Rhodophyta. Em 1996, Edlund \& Wynne (1996) (1996) propuseram o nome Acanthoceras Honigmann para conservação com base no amplo uso do nome e no fato de Acanthoceras Kützing estar sendo ser tratado como sinônimo de Ceramium Roth, proposta esta aceita em 1999 (Compère 1999)(Compère 1999). Um resumo da história taxonômica do gênero Similar história do gênero pode ser encontradoa no estudo de Tremarin et al. (2015).

Posteriormente, verificou-se que $A$. magdeburgense, tipo do gênero Acanthoceras, é idêntico a Attheya zachariasii, sendo tratada, portanto, como sinônimo heterotípico deste último táxon em Acanthoceras (Simonsen 1979, Van Landingham 1967). Dessa forma, Acanthoceras zachariasii passa a ser considerado o nome aceito para o táxon por se tratar de um sinônimo anterior de $A$. magdeburgense. Contudo, é importante considerar que o elemento que continua a tipificar o gênero, é o mesmo que tipifica $A$. magdeburgense, ou seja, a sinonimização não altera o tipo genérico. Dessa forma, caracterizações estruturais e ultraestruturais, a verificação dos locais de ocorrência bem como as características limnológicas e preferências da espécie são primordiais para a taxonomia e conhecimento da ecologia desse gênero.

As localidades de ocorrência de A. zachariasii na região centro-norte do Brasil apresentaram características limnológicas distintas (tabela 2). Os ambientes da Bacia Amazônica foram mais profundos, com águas de temperaturas mais elevadas e maior condutividade e transparência. Enquanto que as águas da Bacia Tocantins-Araguaia apresentaramse mais rasas com a transparência e condutividade menores.

Ludwig \& Tremarim-Bigunas (2006) sugerem que a rara ocorrência de $A$. zachariasii deve-se provavelmente a problemas metodológicos ligados à oxidação e preservação dos espécimes. Apesar dos cuidados com o processo de fixação e a análise de amostras não oxidadas, esta espécie apresentou-se como de rara ocorrência nas regiões estudadas. Das 454 amostras analisadas (Bacia amazônica $n=182$; Bacia Tocantins Araguaia $n=272)$, este táxon foi registrado em apenas 14 destas (3,08\% das amostras). Além disso, o táxon ocorreu com a expressividade muito inferior se comparado às demais espécies, sendo o maior valor de densidade de 62 ind. $\mathrm{mL}^{-1}$ na
Bacia Tocantins-Araguaia e de 73 ind. $\mathrm{mL}^{-1}$ na Bacia Amazônica.

Krammer \& Lange-Bertalot (1991) mencionaram que este táxon está geralmente associado com Urosolenia longiseta (O. Zacharias) Edlund \& Stoermer, o que foi confirmado em $85 \%$ das amostras da bacia Tocantins-Araguaia. No entanto, dentre as amostras analisadas da bacia amazônica, apenas em uma (Am1) foi confirmada esta coocorrência.

Nas duas regiões hidrográficas analisadas neste estudo, Acanthoceras zachariasii foi registrada em ambientes meso a eutróficos. Para a bacia amazônica A. zachariasii ocorreu predominantemente no período de potamofase (i.e., ambientes com maior profundidade), lênticos, e levemente ácidos. Já para a bacia Tocantins-Araguaia, A. zachariasii ocorreu no período de limnofase (i.e., ambientes rasos), lênticos, e pH de levemente ácido a básico. Vários autores também tem registrado a ocorrência deste táxon em sistemas aquáticos com características de trofia similares (Gupta \& Singh 1985, Hustedt 1930, Krammer \& Lange-Bertalot 1991, Lepistö 1999, Lepistö et al. 2004, Napiórkowska-Krzebietke et al. 2011). Em outros casos, esta espécie ocorre tanto em ambientes oligotróficos (Townsend 2006) quanto hipereutróficos (Edlund \& Stoermer 1993), alcalinos, e com elevada transparência (Edlund \& Stoermer 1993, Townsend 2006).

\section{Conclusão}

Acanthoceras zachariasii (Brun) Simonsen é um táxon de rara ocorrência nas regiões hidrográficas Amazônica e Tocantins-Araguaia, em fases de água alta e baixa, respectivamente, bem como no restante do país, sendo registrada principalmente em ambientes lênticos, mesotróficos a eutróficos, levemente ácidos a alcalinos. Um panorama da ocorrência de $A$. zachariasii no Brasil a partir desse estudo é fornecido na figura 8 .

A ampla variabilidade de caracteres métricos observada a partir do material de A. zachariasii do centro-norte brasileiro em relação a outros trabalhos que registram esse táxon, sugerem a necessidade de estudos taxonômicos polifásicos, incluindo dados moleculares e químicos, para melhor circunscrição do táxon e avaliação da representatividade dos caracteres morfológicos utilizados até agora na delimitação da espécie. 


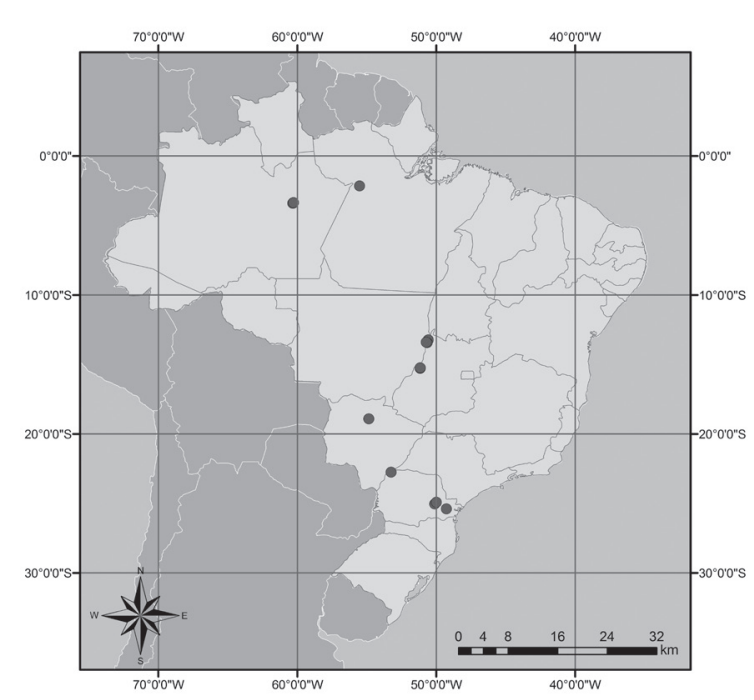

Figura 8. Mapa de ocorrência de Acanthoceras zachariasii (Brun) Simonsen no Brasil, sintetizado a partir deste estudo e dos trabalhos de Shirata (1986), Shirata \& Valente-Moreira (1987), Train \& Rodrigues (2004), Moss \& Moss (2007), e Tremarin et al. (2015).

Figure 8. Map of occurence of Acanthoceras zachariasii (Brun) Simonsen in Brazil, sinthetized from this study and from Shirata (1986), Shirata \& Valente-Moreira (1987), Train \& Rodrigues (2004), Moss \& Moss (2007), and Tremarin et al. (2015).

\section{Agradecimentos}

Os autores agradecem ao Laboratório Multiusuário de Microscopia de Alta Resolução (LabMic - UFG) pela obtenção de imagens. Aos Dr. Gwenael April (Universidade de Bordeaux, França), Coordenador do Projeto Carbono na Amazonia (Carbama), e a Dra. Marie-Pole Bonnet (IRD, França) pelas informações limnológicas e amostras de fitoplâncton oriundas da Bacia Amazônica. R.R. Morais agradece à FAPEG (Fundação de Amparo à Pesquisa do Estado de Goiás) pela concessão de bolsa de mestrado. W.J da Silva agradece à CAPES (Coordenação de Aperfeiçoamento de Pessoal de Nível Superior) por bolsa de pósdoutorado PNPD/CAPES.

\section{Literatura citada}

Abarca, N., Jahn, R., Zimmermann, J. \& Enke, N. 2014. Does the cosmopolitan diatom Gomphonema parvulum (Kützing) Kützing have a biogeography? PLoS ONE 9: e86885.

Agardh, C.A. 1824. Systema algarum. Litteris Berlingianus, Lundae [Lund].

APHA. 2005. Standard methods for examination of water and wastewater. 21 ed. American Public Health Association, Washington.
Beaver, J. 1981. Apparent ecological characteristics of some common freshwater diatoms. Ontario Ministry of the Environment Report, Ontario.

Bicudo, D.C. 1990 Considerações sobre metodologias de contagem de algas do perifíton. Acta Limnologica Brasiliensia 3: 459-475.

Bicudo, C.E.M. \& Menezes, M. 2006. Gêneros de águas continentais do Brasil. Chave para identificação e descrições. 2 ed. Rima, São Carlos.

Brun, J. 1894. Zwei Neue Diatomeen von Ploen. Forsehungsberiehte aus der Biologisehen Station zu Plön 2: 53-56.

Cărăus, I. 2012. Algae of Romania. A distributional checklist of actual algae. Studii si Cercetări - Biologie. Univ. Bacău. 3 rev. 7: 1-809.

Compère, P. 1999. Report of the Committee for Algae: 5. Taxon 48: 129-132.

Dillwyn, L.W. 1809. British Confervae or colored figures and descriptions of the British Plants referred by botanists to the genus Conferva. W. Phillips, London.

Edlund, M. B. \& Stoermer, E.F. 1993. Resting spores of the freshwater diatoms Acanthoceras and Urosolenia. Journal of Paleolimnology 9: 55-61.

Edlund, M.B. \& Wynne, M.J. 1996. Proposal to conserve the name Acanthoceras Honigm. (Bacillariophyceae) against Acanthoceras Kutz. (Rhodophyceae). Taxon 45: 529-530.

Ferrario, M.E., Damborenea, M.C. \& Sar, E.A. 1992. Consideraciones sobre Acanthoceras zachariasii (Bacillariophceae). Darwiniana 31: 357-359.

Genkal, S.I. \& Chekryzhevab, T.A. 2011. Centric Diatoms (Bacillariophyta, Centrophyceae) in Karelian Waterbodies. Inland Water Biology 4: 1-11.

Grasshoff, K. 1983. Methods of seawater analysis. Verlag Chemie, Weinhein.

Gupta, B.P. \& Singh, N.S. 1985. An Interesting Observation on a Monospecific Bloom of a Diatom Attheya zachariasi Brun from a Man. Made Lake. Journal of Inland Fisheries Society of India, 17: 84-86.

Honigmann, H. 1910. Beiträge zur Kenntnis des Süßwasser-planktons. Archiv für Hydrobiologie und Planktonkunde 5: 71-78.

Huber-Pestalozzi, G. 1942. Das Phytoplankton des Süsswassers. Diatomeen. In: A. Thienemann (ed.). Die Binnengewässer, Band 16. Schweizerbart'sche Verlagsbuchhandlung, Stuttgart, pp. 367-549.

Hustedt, F. 1930. Die Kieselalgen Deutschlands: Osterreichs und der Schweiz. In: F. Hustedt (ed.). L. Rabenhorst's Kryptogamen-Flora. Akademische Verlagsgesellschaft, Leipzig, pp. 1-920.

Jahn, R. 2004. Discovery of the type specimen of Pinnularia gastrum Ehrenberg, the type species of the genus Placoneis Mereschkowsky. Diatom Research 19: 229-234. 
Kermarrec, L., Bouchez, A., Rimet, F. \& Humbert, J.F. 2013. First evidence of the existence of semi-cryptic species and of a phylogeographic structure in the Gomphonema parvulum (Kützing) Kützing complex (Bacillariophyta). Protist 164: 686-705.

Krammer, K. \& Lange-Bertalot, H. 1991. Bacillariophyceae: Centrales, Fragilariaceae, Eunotiaceae. In: H. Ettl, J. Gerloff, H. Heyning \& D. Mollenhauer (eds.). Süsswasserflora von Mitteleuropa, Band 2, Teil 3, Gustav Fisher, Stuttgart, pp. 1-576.

Kützing, F.T. 1842. Ueber Ceramium Ag. Linnaea 15: 727-746.

Lepistö, L. 1999. Phytoplankton assemblages reflecting the ecological status of lakes in Finland. Monographs of the Boreal Environment Research, v. 16. Finnish Environment Institute, Helsinki, pp. 5-43.

Lepistö, I.L., Holopainen, A. \& Vuoristo, H. 2004. Typespecific and indicator taxa of phytoplankton as a quality criterion for assessing the ecological status of Finnish boreal lakes. Limnologica 34: 236-248.

Li, Y., Zhou, X., Feng, G., Hu, H., Niu, L., Hebert, P.D. N. \& Huang, D. 2010. COI and ITS2 sequences delimit species, reveal cryptic taxa and host specificity of figassociated Sycophila (Hymenoptera, Eurytomidae). Molecular ecology resources 10: 31-40.

McNeill, J., Barrie, F.R., Buck, W.R., Demoulin, V., Greuter, W., Hawksworth, D.L., Herendeen, P.S., Knapp, S., Marhold, K., Prado, J., Prud'homme Van Reine, W.F., Smith, G.F., Wiersema, J.H. \& Turland, N.J. 2012. International Code of Nomenclature for algae, fungi, and plants (Melbourne Code) adopted by the Eighteenth International Botanical Congress, Melbourne, Australia, July 2011. Regnum Vegetabile 154: 1-240. Koeltz Scientific Books, Königstein.

Lozo, R.N., Berté, S., Komoé, K., Yao, S.S. \& Kouamélan, E.P. 2013. Bacillariophyceae (Heterokontophyta) from Bandama River in Côte d'Ivoire, West Africa. Journal of Animal \& Plant Sciences 20: 3113-3121.

Ludwig, T.A.V. \& Tremarim-Bigunas, P.I. 2006. Bacillariophyta. In: C.E.M. Bicudo \& M. Menezes (eds.). Gêneros de algas de águas continentais do Brasil. Chave para identificação e descrições. 2 ed. Rima, São Carlos, pp. 391-439.

Lund, J.W.G., Kipling, C. \& Lecren, E.D. 1958. The inverted microscope method of estimating algal number and the statistical basis of estimating by counting. Hydrobiologia 11: 143-170.

Mann, D.G. 1999. The species concept in diatoms. Phycological Research 38: 437-495.

Mann, D.G. \& Evans, K.M. 2007. Molecular and the neglected art of diatomics. In: J. Brodie \& J. Lewis (eds.). Unravelling the algae: the past, present, and future of algal systematics. CRC Press, Boca Raton, pp. 231-265.
Margalef, N. 1983. Limnologia. Ediciones Omega, Barcelona.

Moss, G. \& Moss, M. 2007. Relatório Projeto Brasil Das Águas: Sete Rios. Brasília.

Napiórkowska-Krzebietke, A. Hutorowicz, A. \& Tucholski, S. 2011. Dynamics and Structure of Phytoplanktonin Fishponds Fed with Treated Wastewater. Polish Journal of Environmental Studies 20: $157-166$.

Negro, A.I. \& Hoyos, C. 2006. Relationships between diatoms and the environment in Spanish reservoirs. Limnetica 24: 133-144.

Nõges, P. \& Viirret, M. 2001. Environmental conditions and the development of Planktonema lauterbornii Schimidle in phytoplankton of Karhijärvi, a lake in SW Finland. Boreal Environmental Research 6: 181-190.

OECD. 1982. Eutrophication of waters. Monitoring, assessment and control. OECD, Paris.

Rivera, P. 1974. Diatomeas de la Laguna Verde del Parque Hualpén, Chile. Interesantes representantes del gênero Attheya (West, 1860). Boletin de la Sociedad de Biología de Concepción, 47: 87-91.

Round, F.E., Crawford, R.M. \& Mann, D.G. 1990. The Diatoms: biology and morphology of the genera. Cambridge University Press, Cambridge.

Schagerl, M., Drozdowski, I., Angeler, D.G., Hein, T. \& Preiner, S. 2009. Water age - a major factor controlling phytoplankton community structure in are connected dynamic floodplain (Danube, Regelsbrunn, Austria). Journal of Limnology, 68: 274-287.

Simonsen, R. 1979. The diatom system: ideas on phylogeny. Bacillaria 2: 9-71.

Sinada, F. \& Abdel Karim, A.G. 1984. A quantitative study of the phytoplankton in the Blue and White Niles at Khartoum. Hydrobiologia, 110: 47-55.

Shirata, M.T. 1986. Contribuição ao estudo das diatomáceas (Bacillariophyceae) no lago do Parque São Lourenço, Curitiba, estado do Paraná, Brasil. Dissertação de Mestrado, Universidade Federal do Paraná, Curitiba.

Shirata, M.T. \& Valente-Moreira, I.M. 1987. Ocorrência das diatomáceas Attheia zachariasi e Surirella stalagma no lago do Parque São Lourenço, Curitiba, PR, Brasil. Acta Biológica Paranaense, 16: 87-92.

Townsend, S.A. 2006. Hydraulic phases, persistent stratification, and phytoplankton in a tropical floodplain lake (Mary River, northern Australia). Hydrobiologia 556: 163-179.

Train, S. \& Rodrigues, L.C. 2004. Phytoplanktonic Assemblages. In: S.M. Thomaz, A.A. Agostinho \& N.S. Hahn (eds.). The upper Paraná river and its floodplain: physical aspects, ecology and conservation. Backhuys Publ, Leiden, pp. 103-124. 
Tremarin, P.I., Freire, E.G., Algarte, V.M. \& Ludwig, T.A.V. 2015. Acanthoceras and Urosolenia species (Diatomeae) in Subtropical Reservoirs from South Brazil: ultrastructure, distribution and autoecology. Biota Neotropica 15: 1-16.

Uhelinger, V. 1964. Étude statistique des methodes de dénombrement planctonique. Archives des Sciences 17: 121-223.

Utermöhl, H. 1958. Zur vervollkmmung der quantitativen phytoplankton methodik. Internationale Vereinigung für Theoretische und Anwandte Limnologie. Komitee für Limnologische Methoden 9: 1-88.
VanLandingham, S. L. 1967. Catalogue of the fossil and recent genera and species of diatoms and their synonyms. Part I. Acanthoceras through Bacillaria. J. Cramer, Lehre, pp. 1-493.

Villars, M. 1789. Histoire des plants de Dauphiné, III. Société Royale de Médicine, Grenoble.

Zimmermann, J., Jahn, R. \& Gemeinholzer, B. 2011. Barcoding diatoms: evaluation of the V4 subregion on the $18 \mathrm{~S}$ rRNA gene, including new primers and protocols. Organisms Diversity \& Evolution 11: 173-192. 